\title{
Asset Pricing Models and Stock Selection Ability of the Indian Mutual Fund Managers: An Empirical Study of Open-ended Growth Equity Schemes
}

\section{Kamal Gupta}

Assistant Professor at I.T.S Engineering College, Greater Noida, Punjab, India

\begin{abstract}
The paper is devoted to the study of the analysis and forecasting of the possibility of joint investment Fund managers to choose securities for further investment. The methodological tools of the work are presented by models Jensen, Fama \& amp; French and Carhart (which allow to assess the ability to select securities by managers of mutual funds). Empirical estimates of the analysis on three models showed that Indian mutual Fund managers have the ability to choose stocks. The author states that the analysis based on the Jensen model characterizes higher potential opportunities for the selection of securities by the managers of India's joint-stock funds compared to the other two models used in the study. The results of the study can be useful for investors in making investment decisions, in particular in the process of placing their own financial resources in joint investment funds. The paper postulates that investors will be able to choose joint investment schemes in favor of funds, which provide the opportunity to choose securities for investment for more than ten years. The author notes that the key effect of the introduction of such a practice of interaction between investors and funds will be the growth of investor confidence, which will contribute to the accumulation of additional volumes of investments in the joint investment sector. This study is limited only to the schemes of investing their own financial resources, but in the future can be further expanded to the practice of using a wide range of schemes, since the possibility of choosing shares is associated with many financial processes and indicators. Since the study of asset pricing models is a continuous process, the author proposed to study the processes of joint investment in pension funds in the context of assessing the impact of financial indicators such as liquidity, return on investment, profitability.
\end{abstract}

Keywords: investors, funds of collective investment, the ability to stock selection, patterns of growth equity capital.

JEL Classification: M31, O42.

Cite as: Gupta, K. (2019). Asset Pricing Models and Stock Selection Ability of the Indian Mutual Fund Managers: An Empirical Study of Open-ended Growth Equity Schemes. Financial Markets, Institutions and Risks, 3(3), 49-62. http://doi.org/10.21272/fmir.3(3). 49-62.2019.

(C) The Author, 2019. This article is published with open access at Sumy State University.

\section{Introduction}

The stock-selection ability refers to the ability of the mutual fund managers to make diversification based on their knowledge and expertise. The major advantage ofinvesting in the mutual fund schemes is the professional management services of the mutual fund managers. For this purpose, the investorshave to bear some cost in the form of the mutual fund manager's fees. So, it becomes the responsibility of the mutual fund managers to provide abnormal returns as a premium for the risk assumed by the investors. Therefore, this paper is an attempt to measure the stock-selection ability of the mutual fund managers based on various models.

\section{Review of Literature}

The review of the literature on the stock-selection ability models is very mixed and wide. A good number of studies have been conducted for the same. Stock-selection ability of the mutual fund managers measures the ability of the mutual fund managers to diversify the mutual fund portfolio in a way so that the investors 
can earn abnormal returns as a comparison of the fixed investment and self-made portfolios like index funds. The concept of the diversification was more elaborately defined in 1952, in the paper titled "Portfolio Selection" authored by Harry Markowitz. Markowitz defined the relationship between the beliefs and choice of the portfolio according to the expected return and variance of return around that expected return. Basically, he defined the subjective judgment of the investor in mathematically form. After that Sharpe (1964) tried to further elaborate the Markowitz concept and defined the Capital Asset Pricing Model. Jensen $(1968,1969)$ was the first one to make a contribution to the model of the stock-selection ability of the mutual fund managers using the CAPM model adding the abnormal returns (alpha) in the model.He regressed the mutual fund returns against the market index returns. The results of the study revealed that not even a single mutual fundscheme was able to earn abnormal returns. Further, various empirical studies were done by Carlson(1970), John Macdonald(1974), James R.F-Guy(1978), Tye Kim(1978), Ippolito(1989), Ariff and Johnson(1990), Clumby and Glen(1990), Barua and Varma (1991), Cole and IP(1993), Ajah Shah and Susan Thomas(1994), Kaura and Jayadev(1995), M.Jayadev(1996), Triplaraju and Patil (1998). Majority ofstudies found that mutual fund managers had very less stock-selection ability. Also, some researchers found that the stock-selection ability of the mutual fund managers depend upon the benchmark chosen for measuring the mutual fund performance. The research on stock-selection ability does not confined only on the Jensen model. After that, various studies tried to re-modify the Jensen model and explored that the market index alone does not explain the variation in portfolio returns. There are some other variables like size, leverage, earnings/price and book-to-market equity to its market value \{Banz (1981), Bhandari (1988), Basu (1983) and Rosenberg, Reid and Lanstein (1985)\}. Further, Fama and French (1992a, 1992b) found two relevant factors i.e. 'SMB' and 'HML', which jointly explained the role of other four variables. The study also explained certain issues regarding the slope and intercept of the model. Carhart (1997) again added one more variable i.e. 'momentum' (Jaganathan, 1993) in the three factor Fama \& French Model (1993). Momentum factor refers to the strategies of investing stocks where buying stocks and securities that have had high returns over the past three to twelve months, selling those that have had poor returns over the same period.

After extensive review of literature, it has been found that a lot of research has been done on stock-selection ability of mutual fund managers in the developed countries, while very few studies are found in the Indian context. Therefore, this research is an attempt to contribute to the Indian financial literature. Moreover, most of the studies have focused on yearly, monthly or quarterly NAV data. But, this studyhas used daily returns for all kind of portfolios i.e. mutual fund schemes, market index, SMB, HML and WML.

\section{Research Objectives}

a) To evaluate the stock-selection ability of theIndian mutual fund managers on the basis of Jensen Model.

b) To evaluate the stock-selection ability of theIndian mutual fund managers on the basis of Fama \& French Model.

c) To evaluate the stock-selection ability of the mutual fund managers on the basis of Carhart Model.

d) To identify and suggest future scope of research in this area.

\section{Research Methodology}

\subsection{Sample Selection}

There were total 382 schemes as on $31^{\text {st }}$ March, 2003. Out of this, open-ended growth-oriented schemes constituted 115 schemes. Out of 115 schemes there were only 49 schemes which existed during the research period under study as rest of the schemes either merged with the existing schemes or were no more in existence due to poor performance and other such reasons. The NAV (Net Asset Value) has been collected from Capital Line Database and then further matched with Value Research and moneycontrol.com databases, for verification regarding the nature of the scheme and date of the scheme. In addition, index 
funds and sector funds are excluded from the study as they do not come under the direct purview of diversified equity market segment. Mutual fund percentage returns are measured on a daily basis by using the formula $\operatorname{Ln}(\mathrm{P} 1 / \mathrm{P} 0)^{*} 100$ where $\mathrm{P} 1$ is the current daily's price. $\mathrm{P} 0$ is previous daily's price. The main advantage of using logarithmic returns is that it is not affected by the base effect problem.

\subsection{Benchmark Selection:}

As per SEBI Master Circulation 2013 specific benchmarks have been mentioned for different kinds of mutual fund schemes based on their objectives. In the present study, broad-based index i.e. BSE 500 has been used as this study covers open-ended growth equity schemes.

\subsection{Selection of Risk-free Interest Rate:}

91-day treasury bills have been taken from the RBI data-base as risk-free interest rate, which is available on the annual-rate basis. It has been further calculated on daily basis.

\subsection{Research Methodology}

Three commonly used methods for measuring the stock-selection ability of the mutual fund managers are as follows:

a) Jensen Model: Jensen Model defines the stock-selection ability by regressing the mutual fund returns with market index returns. It can be calculated as:

$\alpha^{J}=\left(R P_{t}-R F_{t}\right)-\beta_{m}\left(R M_{t}-R F_{t}\right)$

Where,

$\alpha^{J}=$ Stock-selection ability of the mutual fund manager

$\mathrm{RP}_{\mathrm{t}}=$ Mutual fund returns during period $\mathrm{t}$.

$\mathrm{RM}_{\mathrm{t}}=$ Market index returns during period $\mathrm{t}$.

$\mathrm{RF}_{\mathrm{t}}=$ Risk-free rate during period $\mathrm{t}$.

$\beta_{m}=$ Systematic Risk from market index

b) The Fama French three-factor model:

$\alpha^{F F}=\left(R P_{t}-R F_{t}\right)-\beta_{m}\left(R M_{t}-R F_{t}\right)-\beta_{S M B} S M B_{t}-\beta_{H M L} H M L_{t}$

Where,

$\alpha^{F F}=$ Stock-selection ability of the mutual fund manager

$\mathrm{RP}_{\mathrm{t}}=$ Mutual fund returns during period $\mathrm{t}$.

$\mathrm{RM}_{\mathrm{t}}=$ Market index returns during period $\mathrm{t}$.

$\mathrm{RF}_{\mathrm{t}}=$ Risk-free rate during period $\mathrm{t}$.

$S M B=$ Premium of small stocks over the big-stocks

$H M F$ Premium of growth stocks over value stocks

$\beta_{m}=$ Systematic Risk from market index 
Financial Markets, Institutions and Risks, Volume 3, Issue 3, 2019

ISSN (online) - 2521-1242 ISSN (print) - 2521-1250

$\beta_{S M \bar{B}}$ Systematic risk of SMB

$\beta_{\text {HML }}=$ Systematic risk of HML

The intercept in this model is referred to as the "three-factor alpha"

c) The Carhart Four-factor Model:

$\alpha^{c}=\left(R P_{t}-R F_{t}\right)-\beta_{m}\left(R M_{t}-R F_{t}\right)-\beta_{S M B} S M B_{t}-\beta_{H M L} H M L_{t}-\beta_{W M L} W M L_{t}$

Where,

$\alpha^{c}=$ Stock-selection ability of the mutual fund manager

$\mathrm{RP}_{\mathrm{t}}=$ Mutual fund returns during period $\mathrm{t}$.

$\mathrm{RM}_{\mathrm{t}}=$ Market index returns during period $\mathrm{t}$.

$\mathrm{RF}_{\mathrm{t}}=$ Risk-free rate during period $\mathrm{t}$.

$S M B=$ Premium of small stocks over the big-stocks

$H M F=$ Premium of growth stocks over value stocks

$W M F=$ Premium of winner stocks over loser stocks

$\beta_{m}=$ Systematic Risk from market index

$\beta_{S M \bar{B}}$ Systematic risk of SMB

$\beta_{\text {HML }}=$ Systematic risk of HML

$\beta_{W M L}=$ Systematic risk of WML

In all the above models, a positive and statistically significant 'alpha' represents the stock-selection ability of the mutual fund managers.

\section{Empirical Results and Interpretation}

\subsection{Descriptive Statistics of Mutual Fund Returns}

Table 5.1 (see in Appendix) explains the descriptive statistics of the mutual fund returns. Daily mean Mutual fund returns ranges from $0.0021 \%$ to $0.1095 \%$. Top five schemes in terms of average mutual fund returns are Reliance Growth Fund (0.109\%), HDFC Top 200 Fund (0.102\%), HDFC Equity Fund (0.100\%), Tata Pure Equity Fund (0.098\%) and Sundarm Select Midcap Fund (0.104\%). Standard Deviation ranges from the $1.1 \%$ to $2.7 \%$. Highest standard deviation is for the Taurus Star share Fund (2.34\%), UTI Top 100 Fund (2.07), UTI Equity Fund (1.93\%), JM Basic Fund (1.91\%) and Escorts Growth Plan (2.69\%). The five least volatile funds as per standard deviation of daily returns are Birla Sunlife Buy India Fund (1.34\%), UTI MNC Fund (1.109\%), Birla Sunlife MNC Fund (1.108\%), HDFC Capital Builder Fund (0.0970\%) and Birla Sunlife Dividend Yield Plus (1.311\%). 
Table 5.1. Descriptive Statistics of MF Returns

\begin{tabular}{|c|c|c|c|c|c|c|c|c|c|}
\hline Mutual Fund Name & $\begin{array}{c}\text { Daily } \\
\text { Mean } \\
\text { Return } \\
(\%) \\
\end{array}$ & Median & $\begin{array}{l}\text { S.D } \\
(\%)\end{array}$ & $\begin{array}{c}\text { Excess } \\
\text { Kurtosis } \\
\end{array}$ & Skewness & Range & Minimum & Maximum & Count \\
\hline Birla Sunlife Advantage Fund & 0.0750 & 0.1660 & 1.61 & 9.98 & -0.31 & 29.46 & -12.61 & 16.85 & 2472 \\
\hline Birla Sunlife Buy India Fund & 0.0964 & 0.1547 & 1.34 & 9.20 & -0.33 & 24.17 & -10.29 & 13.88 & 2472 \\
\hline Birla Sunlife Dividend Yield Plus & 0.0880 & 0.1733 & 1.31 & 9.96 & -0.86 & 21.97 & -11.14 & 10.83 & 2470 \\
\hline Birla Sunlife Equity Fund & 0.0942 & 0.1920 & 1.54 & 9.10 & -0.25 & 27.31 & -11.13 & 16.18 & 2471 \\
\hline Birla Sunlife Frontline Equity Fund & 0.0909 & 0.1620 & 1.44 & 9.34 & -0.15 & 25.10 & -9.72 & 15.38 & 2471 \\
\hline Birla Sunlife Midcap Fund & 0.0968 & 0.2089 & 1.45 & 15.95 & -0.01 & 30.01 & -11.36 & 18.64 & 2471 \\
\hline Birla Sunlife MNC Fund & 0.0887 & 0.1403 & 1.11 & 6.73 & -0.76 & 15.97 & -7.85 & 8.12 & 2472 \\
\hline Birla Sunlife opportunities Fund & 0.0584 & 0.1306 & 1.42 & 7.34 & -0.68 & 20.58 & -10.55 & 10.03 & 2466 \\
\hline DSP BR Opportunities Fund & 0.0939 & 0.1804 & 1.46 & 6.62 & -0.44 & 22.77 & -10.29 & 12.48 & 2473 \\
\hline DWS Alpha Equity Fund & 0.0841 & 0.1599 & 1.53 & 6.02 & -0.43 & 22.75 & -10.66 & 12.09 & 2459 \\
\hline Escorts Growth Plan & 0.0682 & 0.1428 & 2.69 & 607.09 & 0.89 & 157.97 & -77.71 & 80.26 & 2455 \\
\hline Franklin Bluechip India Fund & 0.0944 & 0.1389 & 1.46 & 6.26 & -0.16 & 23.23 & -9.78 & 13.45 & 2467 \\
\hline Franklin India Prima Fund & 0.0968 & 0.1921 & 1.42 & 9.65 & -0.57 & 24.80 & -10.68 & 14.12 & 2464 \\
\hline Franklin India Prima Plus Fund & 0.0953 & 0.1687 & 1.38 & 7.69 & -0.20 & 22.82 & -8.90 & 13.92 & 2463 \\
\hline HDFC Capital Builder Fund & 0.0970 & 0.1865 & 1.34 & 6.97 & -0.65 & 19.98 & -8.53 & 11.45 & 2470 \\
\hline HDFC Equity Fund & 0.1008 & 0.1667 & 1.51 & 6.43 & -0.26 & 24.65 & -10.44 & 14.20 & 2473 \\
\hline HDFC Growth Fund & 0.0953 & 0.1941 & 1.42 & 7.10 & -0.46 & 23.27 & -10.49 & 12.79 & 2470 \\
\hline HDFC Top 200 Fund & 0.1024 & 0.1807 & 1.51 & 6.80 & -0.27 & 25.70 & -11.49 & 14.21 & 2473 \\
\hline HSBC Equity Fund & 0.0915 & 0.1553 & 1.45 & 5.68 & -0.41 & 22.22 & -10.52 & 11.70 & 2471 \\
\hline ICICI Pru Dynamic Plan & 0.0957 & 0.1565 & 1.39 & 6.09 & -0.68 & 18.30 & -9.56 & 8.75 & 2471 \\
\hline ICICI Pru Top 100 & 0.0839 & 0.1483 & 1.56 & 6.52 & -0.32 & 24.57 & -11.17 & 13.40 & 2473 \\
\hline ICICI Top Pru Top 200 & 0.0841 & 0.1471 & 1.54 & 6.64 & -0.34 & 24.57 & -11.17 & 13.40 & 2473 \\
\hline ING Core Equity Fund & 0.0713 & 0.1681 & 1.68 & 13.87 & -0.57 & 30.32 & -15.36 & 14.96 & 2470 \\
\hline JM Basic Fund & 0.0021 & 0.1249 & 1.91 & 14.40 & -1.01 & 39.81 & -20.07 & 19.74 & 2463 \\
\hline JM Equity Fund & 0.0622 & 0.1587 & 1.67 & 10.06 & -0.04 & 30.00 & -11.13 & 18.87 & 2468 \\
\hline Kotak 50 & 0.0376 & 0.1410 & 1.71 & 49.39 & -3.37 & 43.62 & -30.15 & 13.47 & 2470 \\
\hline LIC Nimura Equity Fund & 0.0623 & 0.1500 & 1.69 & 9.87 & -0.35 & 30.57 & -14.02 & 16.55 & 2429 \\
\hline LIC Nomura Growth Fund & 0.0728 & 0.1465 & 1.68 & 11.21 & -0.06 & 30.18 & -11.77 & 18.41 & 2426 \\
\hline Morgan Stanley Growth Fund & 0.0687 & 0.1517 & 1.53 & 10.44 & -0.38 & 28.68 & -12.89 & 15.79 & 2469 \\
\hline Principal Growth Fund & 0.0685 & 0.1618 & 1.47 & 5.71 & -0.57 & 21.18 & -9.53 & 11.65 & 2463 \\
\hline Relaince Growth Fund & 0.1095 & 0.2346 & 1.44 & 7.84 & -0.59 & 23.92 & -10.50 & 13.42 & 2466 \\
\hline Relaince Vision Fund & 0.0878 & 0.1895 & 1.49 & 6.79 & -0.32 & 25.07 & -11.42 & 13.64 & 2466 \\
\hline SBI Contra Fund & 0.0713 & 0.1935 & 1.77 & 46.79 & -3.51 & 40.68 & -25.86 & 14.83 & 2435 \\
\hline Sundram Growth Fund & 0.0797 & 0.1792 & 1.60 & 8.80 & -0.45 & 29.14 & -13.58 & 15.56 & 2469 \\
\hline Sundram Midcap Fund & 0.1036 & 0.1225 & 1.39 & 12.60 & -0.38 & 28.18 & -11.92 & 16.26 & 2660 \\
\hline Sundram Select Focus Fund & 0.0826 & 0.1507 & 1.57 & 11.70 & -0.00 & 31.25 & -12.74 & 18.51 & 2469 \\
\hline Tata Bonaza Fund & 0.0599 & 0.1383 & 1.78 & 26.62 & -1.69 & 43.37 & -26.16 & 17.22 & 2463 \\
\hline Tata Discovery Fund & 0.0616 & 0.2173 & 1.86 & 9.47 & -0.25 & 31.68 & -13.60 & 18.08 & 2471 \\
\hline Tata Equity Opportunities Fund & 0.0981 & 0.1797 & 1.53 & 7.92 & -0.38 & 25.94 & -10.89 & 15.06 & 2463 \\
\hline
\end{tabular}


Table 5.1. (cont.). Descriptive Statistics of MF Returns

\begin{tabular}{|l|r|r|r|r|r|r|r|r|r|}
\hline Tata Ethical Fund & 0.0921 & 0.1651 & 1.50 & 7.15 & -0.81 & 21.77 & -11.43 & 10.34 & 2463 \\
\hline Tata Growth Fund & 0.0854 & 0.1759 & 1.59 & 10.39 & -0.29 & 25.25 & -10.85 & 14.40 & 2269 \\
\hline Tata Pure Equity Fund & 0.0983 & 0.1675 & 1.48 & 7.96 & -0.28 & 24.61 & -10.01 & 14.59 & 2465 \\
\hline Tata Starshare Fund & 0.0949 & 0.1516 & 2.34 & 202.61 & 0.33 & 104.53 & -51.34 & 53.19 & 2471 \\
\hline UTI Equity Fund & 0.0762 & 0.1614 & 1.93 & -1.90 & 31.39 & -20.43 & 10.96 & 187.37 & 2459 \\
\hline UTI Master Plus & 0.0702 & 0.1665 & 1.55 & 7.67 & -0.31 & 25.68 & -10.85 & 14.83 & 2465 \\
\hline UTI Master Value Fund & 0.0442 & 0.2084 & 1.79 & 175.39 & -8.53 & 51.27 & -41.38 & 9.89 & 2465 \\
\hline UTI MNC Fund & 0.0797 & 0.1349 & 1.11 & 12.40 & -1.31 & 19.36 & -12.39 & 6.97 & 2465 \\
\hline UTI Top 100 Fund & 0.0323 & 0.1467 & 2.07 & 486.29 & -15.10 & 82.90 & -68.43 & 14.46 & 2464 \\
\hline
\end{tabular}

Note: Listed are average summary statistics of the 49 mutual funds in the sample and the market index. The Sample period is April 1 , 2003, to March 31, 2013, a total of 2742 trading days or 120 trading months. The mean $(\mu)$ and standard deviation $(\sigma)$ are sample estimates. Skewness is computed as $\mathrm{S}=1 / \sigma 3 \mathrm{~T} \sum_{t=1}^{T}(R t \quad \mu) 3$,And excess kurtosis $(\mathrm{K})$ is computed as $\mathrm{K}=1 / \sigma 3 \mathrm{~T} \sum_{t=1}^{T}(R t-\mu) 4-3$.

Table 5.2. Descriptive Statistics of Mutual Fund Returns, Market Index Return, SMB, HML and WML

\begin{tabular}{|c|c|c|c|c|c|c|c|c|}
\hline & Daily Mean & Median & S.D & Kurtosis & Skewness & Range & Minimum & Maximum \\
\hline Rp & 0.0784 & 0.1643 & 1.61 & 45.59 & -0.33 & 32.82 & -16.79 & 20.81 \\
\hline Rm & 0.0761 & 0.1969 & 1.62 & 8.78 & -0.42 & 28.20 & -12.44 & 15.76 \\
\hline SMB & 0.0321 & 0.0669 & 0.91 & 5.39 & -0.51 & 14.21 & -8.51 & 5.71 \\
\hline HML & 0.0406 & 0.0275 & 0.92 & 3.26 & -0.16 & 11.27 & -6.09 & 5.18 \\
\hline WML & 0.0657 & 0.1079 & 0.93 & 6.83 & -0.24 & 15.30 & -6.52 & 8.78 \\
\hline
\end{tabular}

Source: Data Compiled by the Researcher.

Table -5.2 explains the descriptive statistics of the variables mutual fund schemes returns, market index returns, SMB, HML and WML. It is evident from table 5.2 that average mutual fund return (0.0784) is the highest among all other portfolios like market index giving an average return (0.0761), SMB with an average return of (0.0321), HML providing an average return of (0.0406) and WML giving an average return of $(0.0657)$. At the same time this can be seen that volatility of mutual fund returns $(1.61 \%)$ and market index returns (1.62) are approximately same. Further, the volatility of SMB (S.D-0.91\%), HML (S.D-0.92\%) and WML(S.D.-0.93\%) are almost equal. Furthermore, mutual funds exhibit higher excess kurtosis than the market index, which is due very high kurtosis of the mutual fund scheme of Escorts Growth Fund (607.13). The market index shows larger negative scenes as compared to the mutual fund schemes. The negative skewness is probably due to the global recession of 2008 and Euro crisis of 2009.

Table 5.3 Correlation Matrix

\begin{tabular}{|c|c|c|c|c|}
\hline Variables & (Rm-Rf)\% & SMB\% & HML\% & WML\% \\
\hline (Rm-Rf) $\%$ & 1.000 & -0.408 & 0.068 & -0.177 \\
\hline SMB\% & -0.408 & 1.000 & 0.340 & -0.012 \\
\hline HML\% & 0.068 & 0.340 & 1.000 & -0.036 \\
\hline WML\% & -0.177 & -0.012 & -0.036 & 1.000 \\
\hline
\end{tabular}

Source: Data Compiled by the Researcher.

Table 5.3 reports the correlation matrix for the independent variables used in the Jensen model, Fama \& French Model and Carhart Model. The correlation between the SMB and WML factors is negative, which is consistent with the Carhart (1997). Low correlation between the independent variables overcomes the possibility of the multicollinearity.

\subsection{Jensen Model (1968)- Empirical Results}

Table 5.4 represents the alpha, the $\mathrm{t}$-value and the values of the coefficient of determination (Adjusted- $\mathrm{R}^{2)}$ for every mutual fund in the sample. 
Table 5. 4. Jensen Model Results for Open-ended Growth Equity Schemes

\begin{tabular}{|c|c|c|c|c|c|c|}
\hline S.No. & Mutual Fund Scheme & Intercept & $t$ Stat & Beta & $t$ Stat & $\begin{array}{c}\text { Adjusted R } \\
\text { Square }\end{array}$ \\
\hline 1 & Birla Sunlife Advantage Fund & 0.001 & 0.09 & $0.966 *$ & 189.74 & 0.936 \\
\hline 2 & Birla Sunlife Buy India Fund & $0.034 *$ & 2.67 & $0.732 *$ & 92.79 & 0.777 \\
\hline 3 & Birla Sunlife Dividend Yield Plus & $0.026^{*}$ & 2.28 & $0.734 *$ & 105.79 & 0.819 \\
\hline 4 & Birla Sunlife Equity Fund & $0.023 *$ & 2.78 & $0.915^{*}$ & 182.22 & 0.931 \\
\hline 5 & Birla Sunlife Frontline Equity Fund & $0.022 *$ & 3.39 & $0.869^{*}$ & 221.52 & 0.952 \\
\hline 6 & Birla Sunlife Mid Cap Fund & $0.030 *$ & 2.46 & $0.814 *$ & 106.77 & 0.822 \\
\hline 7 & Birla Sunlife MNC Fund & $0.033 *$ & 3.05 & $0.597 *$ & 88.49 & 0.760 \\
\hline 8 & Birla Sunlife Opportunities Fund & -0.006 & -0.49 & $0.779 *$ & 96.60 & 0.791 \\
\hline 9 & DSP BR Opportunities Fund & $0.024 *$ & 3.97 & $0.881 *$ & 235.27 & 0.957 \\
\hline 10 & DWS Alpha Equity Fund & 0.013 & 1.56 & $0.906^{*}$ & 170.38 & 0.922 \\
\hline 11 & Escorts Growth Plan & 0.035 & 0.65 & $0.152 *$ & 4.56 & 0.008 \\
\hline 12 & Franklin India Bluechip Fund & $0.024 *$ & 3.38 & $0.87 *$ & 192.30 & 0.937 \\
\hline 13 & Franklin India Prima Fund & $0.034 *$ & 2.39 & $0.752 *$ & 87.94 & 0.758 \\
\hline 14 & Franklin India Prima Plus & $-0.0034 *$ & -26.52 & $0.849 *$ & 196.98 & 0.940 \\
\hline 15 & HDFC Capital Builder Fund & $0.064 *$ & 2.42 & $0.156^{*}$ & 9.53 & 0.035 \\
\hline 16 & HDFC Equity Fund & $0.030^{*}$ & 3.37 & $0.891 *$ & 159.77 & 0.912 \\
\hline 17 & HDFC Growth Fund & $0.027^{*}$ & 3.53 & $0.842 *$ & 175.87 & 0.926 \\
\hline 18 & HDFC Top 200 Fund & $0.031 *$ & 4.66 & $0.909^{*}$ & 220.54 & 0.952 \\
\hline 19 & HSBC Equity Fund & $0.023 *$ & 3.16 & $0.866^{*}$ & 190.35 & 0.936 \\
\hline 20 & ICICI Pru Dynamic Plan & $0.061 *$ & 2.25 & $0.188^{*}$ & 11.20 & 0.048 \\
\hline 21 & ICICI Pru Top 100 & 0.049 & 1.59 & $0.199 *$ & 10.48 & 0.042 \\
\hline 22 & ICICI Pru Top 200 & 0.012 & 1.57 & $0.925 *$ & 196.49 & 0.940 \\
\hline 23 & ING Core Equity Fund & 0.036 & 1.09 & $0.201^{*}$ & 9.76 & 0.037 \\
\hline 24 & JM Basic Fund & 0.000 & -0.70 & $1.02 *$ & 97.30 & 0.793 \\
\hline 25 & JM Equity Fund & -0.013 & -1.27 & $0.983 *$ & 155.15 & 0.907 \\
\hline 26 & Kotak 50 & -0.033 & -1.85 & $0.897 *$ & 80.80 & 0.726 \\
\hline 27 & LIC Nomura Growth Fund & -0.003 & -0.22 & $0.963 *$ & 130.02 & 0.875 \\
\hline 28 & LIC Noumra Equity Fund & -0.015 & -1.54 & $0.995^{*}$ & 169.17 & 0.922 \\
\hline 29 & Morgan Stanley Growth Fund & -0.003 & -0.43 & $0.917 *$ & 203.62 & 0.944 \\
\hline 30 & Principal Growth Fund & -0.001 & -0.19 & $0.877 *$ & 185.72 & 0.933 \\
\hline 31 & Relaince Vision Fund & $0.018^{*}$ & 2.23 & $0.885^{*}$ & 173.14 & 0.924 \\
\hline 32 & Reliance Growth Fund & $0.042 *$ & 4.51 & $0.841 *$ & 145.28 & 0.895 \\
\hline 33 & SBI Contra Fund & -0.001 & -0.05 & $0.908 *$ & 73.39 & 0.689 \\
\hline 34 & Sundram Growth Fund & 0.006 & 0.74 & $0.956^{*}$ & 194.73 & 0.939 \\
\hline 35 & Sundram Midcap Fund & $0.042 *$ & 3.51 & $0.802 *$ & 104.75 & 0.805 \\
\hline 36 & Sundram Select Focus Fund & 0.010 & 1.08 & $0.925^{*}$ & 155.76 & 0.908 \\
\hline 37 & Tata Bonaza Fund & -0.018 & -1.07 & $0.977 *$ & 95.53 & 0.787 \\
\hline 38 & Tata Discovery Fund & -0.013 & -0.69 & $0.979 *$ & 81.31 & 0.728 \\
\hline 39 & Tata Equity Opportunities Fund & $0.027 *$ & 2.84 & $0.898^{*}$ & 153.25 & 0.905 \\
\hline 40 & Tata Ethical Fund & $0.024 *$ & 2.13 & $0.858 *$ & 123.75 & 0.861 \\
\hline
\end{tabular}


Table 5. 4. (cont.). Jensen Model Results for Open-ended Growth Equity Schemes

\begin{tabular}{|c|l|c|c|c|c|c|}
\hline 41 & Tata Growth Fund & 0.012 & 0.93 & $0.845^{*}$ & 109.81 & 0.842 \\
\hline 42 & Tata Pure Equity Fund & $0.029^{*}$ & 3.76 & $0.880^{*}$ & 184.14 & 0.932 \\
\hline 43 & Tatastarshare Fund & 0.020 & 0.57 & $0.983^{*}$ & 46.02 & 0.461 \\
\hline 44 & UTI Equity Fund & 0.010 & 1.00 & $0.800^{*}$ & 128.25 & 0.870 \\
\hline 45 & UTI Master Plus & -0.002 & -0.31 & $0.929^{*}$ & 193.95 & 0.939 \\
\hline 46 & UTI Master Share & -0.002 & -0.18 & $0.833^{*}$ & 146.81 & 0.898 \\
\hline 47 & UTI Master Value Fund & -0.020 & -0.77 & $0.765^{*}$ & 47.55 & 0.478 \\
\hline 48 & UTI MNC Fund & $0.025^{*}$ & 2.10 & $0.582^{*}$ & 79.22 & 0.718 \\
\hline 49 & UTI Top 100 Fund & -0.038 & -1.24 & $0.881^{*}$ & 46.83 & 0.471 \\
\hline
\end{tabular}

Source: Data Compiled by the Researcher.

Note: “*” represent significant at $5 \%$.

Out of the fourty nine mutual funds, Twenty-Two schemes have a positive $\alpha$. The schemes having positive significant alpha at 5\% level of significance are Birla Sunlife Buy India Fund (0.034), Birla Sunlife Dividend Yield Plus (0.026), Birla Sunlife Equity Fund(0.023), Birla Sunlife Frontlife Equity Fund (0.022), Birla Sunlife Mid cap Fund(0.030), Birla Sunlife MNC Fund(0.033), DSP BR opportunities Fund (0.024), Franklin India Bluechip Fund(0.024), Franklin India Prima Fund (0.034), Franklin India Prima Plus (0.0034), HDFC Capital Builder Fund (0.064), HDFC Equity Fund (0.030), HDFC Growth Fund (0.027), HDFC Top 200 Fund (0.031), HSBC Equity Fund (0.023), ICICI Pru Dynamic Plan (0.061), Reliance Vision Fund (0.018), Reliance Growth Fund (0.042), Sundaram Midcap Fund (0.042), Tata Equity Opportunities Fund (0.027), Tata Ethical Fund (0.024), Tata Pure Equity Fund (0.029) and UTI MNC Fund(0.025). There is only one scheme having negative significant value i.e. Franklin India Prima Plus (0.0034). Rest of the 25 schemes is having insignificant value both positive and negative. It is also noticeable that the adjusted $\mathrm{R}^{2}$ is quite high for most of the funds, which mean that market index contribute a major role in explaining the variation among the mutual fund returns. It is evident from the above table that, almost all the domestic equity schemes used for the study are showing adjusted-R square greater than $50 \%$ except only a few schemes i.e. Escorts Growth Plan(0.008), HDFC Capital Builder Fund (0.035), ING Core Equity Fund(0.037), ICICI Pru Top 100(0.042), ICICI Pru Dynamic Plan (0.048), Taurus starshare Fund (0.461), UTI Top 100 Fund (0.471) and UTI Master Value Fund (0.478).

\subsection{Fama and French Model- Empirical Results}

Table 5.5 reports the results for the Fama \& French model.30.61\% of the sample schemes (15 out of the 49) are having statistically significant selectivity on the basis of the Fama \&French Model, using daily data. The schemes rejecting null hypothesis (i.e. there is negative or no stock-selection ability) are Birla Equity Fund (0.018), Birla Sunlife Frontline Equity Fund (0.023), Birla Sunlife MNC Fund (0.031), DSP BR Opportunities Fund (0.023), Franklin India Bluechip Fund (0.030), HDFC Equity Fund(0.028), HDFC Growth Fund(0.024), HDFC Top 200 Fund(0.035), HSBC Equity Fund (0.025), ICICI Pru Top 200 Fund(0.019), HDFC Growth Fund (0.029), Sundaram Select Midcap Fund (0.0235) and Tata Pure Equity Fund (0.028). The schemes having adjusted R-square less than 50\% are Escorts Growth Plan (0.018), HDFC Capital Builder Fund (0.085), ICICI Pru Dynamic Plan (0.086), ICICI Pru Top 100 Fund (0.061), ING Core Equity Fund (0.059), Taurus Starshare Fund (0.475) and UTI Master Value Fund (0.50).

\subsection{Carhart Model- Empirical Results}

The results show that out of the 49 equity schemes, only 15 schemes are having positive stock-selection ability at the significant level i.e. around $30.61 \%$. The Schemes having the positive, significant stockselection ability are Birla Sunlife Buy India Fund (0.024), Birla Sunlife Equity Fund (0.017), Birla Sunlife Frontline Equity Fund (0.034), Birla Sunlife MNC Fund (0.030), DSP BR opportunities Fund (0.017), 
Franklin India Bluechip Fund (0.0290), HDFC Equity Fund (0.029), HDFC Growth Fund (0.021), HDFC Top 200 Fund (0.034), HSBC Equity Fund(0.018), ICICI Pru Top 200 (0.015), JM Basic Fund (.488), Reliance Growth Fund (0.028), Sundaram Select Midcap Fund (0.026) and Tata Pure Equity Fund (0.020).

Table 5.7 reports the comparison of R-square of the Jensen Model, Fama\& French Model and Carhart model. Adjusted R-Square explains the variation of the independent variables (i.e. market index returns, SMB, HML and WML) with the dependent variable (i.e. mutual fund returns). The comparison indicates that Jensen and Fama \& French Models are much more relevant in the Indian financial markets as compared to the Carhart Model. That means thatWML variable have very less role to explain the variation among mutual fund returns at daily prices. On daily returns momentum strategy to get premium has very less role as compared to the SMB and HML Variables.

Table 5.8. Comparison of Adjusted R-square of Jensen, Fama\& French and Carhart Models

\begin{tabular}{|c|c|c|c|c|}
\hline S.No. & Mutual Fund Name & Jensen Model & Fama\& French Model & Carhart Model \\
\hline 1 & Birla Sunlife Advantage Fund & 0.94 & 0.94 & 0.94 \\
\hline 2 & Birla Sunlife Buy India Fund & 0.78 & 0.80 & 0.80 \\
\hline 3 & Birla Sunlife Dividend Yield Plus & 0.82 & 0.84 & 0.85 \\
\hline 4 & Birla Sunlife Equity Fund & 0.93 & 0.93 & 0.93 \\
\hline 5 & Birla Sunlife Frontline Equity Fund & 0.95 & 0.95 & 0.95 \\
\hline 6 & Birla Sunlife Mid Cap Fund & 0.82 & 0.86 & 0.86 \\
\hline 7 & Birla Sunlife MNC Fund & 0.76 & 0.76 & 0.76 \\
\hline 8 & Birla Sunlife Opportunities Fund & 0.79 & 0.81 & 0.81 \\
\hline 9 & DSP BR Opportunities Fund & 0.96 & 0.96 & 0.96 \\
\hline 10 & DWS Alpha Equity Fund & 0.92 & 0.92 & 0.93 \\
\hline 11 & Escorts Growth Plan & 0.01 & 0.02 & 0.02 \\
\hline 12 & Franklin India Bluechip Fund & 0.94 & 0.94 & 0.94 \\
\hline 13 & Franklin India Prima Fund & 0.76 & 0.83 & 0.83 \\
\hline 14 & Franklin India Prima Plus & 0.94 & 0.94 & 0.94 \\
\hline 15 & HDFC Capital Builder Fund & 0.04 & 0.08 & 0.10 \\
\hline 16 & HDFC Equity Fund & 0.91 & 0.91 & 0.91 \\
\hline 17 & HDFC Growth Fund & 0.93 & 0.93 & 0.93 \\
\hline 18 & HDFC Top 200 Fund & 0.95 & 0.95 & 0.95 \\
\hline 19 & HSBC Equity Fund & 0.94 & 0.94 & 0.94 \\
\hline 20 & ICICI Pru Dynamic Plan & 0.05 & 0.09 & 0.10 \\
\hline 21 & ICICI Pru Top 100 & 0.04 & 0.06 & 0.07 \\
\hline 22 & ICICI Pru Top 200 & 0.94 & 0.95 & 0.95 \\
\hline 23 & ING Core Equity Fund & 0.04 & 0.06 & 0.06 \\
\hline 24 & JM Basic Fund & 0.80 & 0.80 & 0.80 \\
\hline 25 & JM Equity Fund & 0.91 & 0.91 & 0.91 \\
\hline 26 & Kotak 50 & 0.73 & 0.73 & 0.73 \\
\hline 27 & LIC Nomura Growth Fund & 0.87 & 0.87 & 0.87 \\
\hline 28 & LIC Noumra Equity Fund & 0.92 & 0.92 & 0.92 \\
\hline 29 & Morgan Stanley Growth Fund & 0.94 & 0.94 & 0.94 \\
\hline 30 & Principal Growth Fund & 0.93 & 0.94 & 0.94 \\
\hline 31 & Relaince Vision Fund & 0.92 & 0.93 & 0.93 \\
\hline 32 & Reliance Growth Fund & 0.90 & 0.92 & 0.92 \\
\hline
\end{tabular}


Table 5.8. (cont.). Comparison of Adjusted R-square of Jensen, Fama\& French and Carhart Models

\begin{tabular}{|c|l|c|c|c|}
\hline 33 & SBI Contra Fund & 0.69 & 0.69 & 0.69 \\
\hline 34 & Sundram Growth Fund & 0.94 & 0.94 & 0.94 \\
\hline 35 & Sundram Select Midcap Fund & 0.80 & 0.86 & 0.86 \\
\hline 36 & Sundram Select Focus Fund & 0.91 & 0.79 & 0.91 \\
\hline 37 & Tata Bonaza Fund & 0.79 & 0.78 & 0.79 \\
\hline 38 & Tata Discovery Fund & 0.73 & 0.92 & 0.92 \\
\hline 39 & Tata Equity Opportunities Fund & 0.91 & 0.88 & 0.88 \\
\hline 40 & Tata Ethical Fund & 0.86 & 0.88 & 0.88 \\
\hline 41 & Tata Growth Fund & 0.84 & 0.93 & 0.94 \\
\hline 42 & Tata Pure Equity Fund & 0.93 & 0.48 & 0.48 \\
\hline 43 & Taurus starshare Fund & 0.46 & 0.87 & 0.87 \\
\hline 44 & UTI Equity Fund & 0.87 & 0.94 & 0.94 \\
\hline 45 & UTI Master Plus & 0.94 & 0.90 & 0.90 \\
\hline 46 & UTI Master Share & 0.90 & 0.50 & 0.50 \\
\hline 47 & UTI Master Value Fund & 0.48 & 0.74 & 0.75 \\
\hline 48 & UTI MNC Fund & 0.72 & 0.47 & 0.47 \\
\hline 49 & UTI Top 100 Fund & 0.47 & & \\
\hline
\end{tabular}

Source: Data Compiled by the Researcher.

\section{Conclusion}

This paper focused on identifying the stock-selection ability of the Indian mutual fund managers on the basis of Jensen (1968), Fama\& French (1993) and Carhart (1997) models. The results of Jensen Model revealed that $46.94 \%$ (23 out of 49 ) mutual fund schemes had stock selection ability while $53.06 \%$ (26 out of 49) schemes did not perform well due to lack of stock selection ability among mutual fund managers. On the basis of Fama \& French Model, it was found that 30.61\% (15 out of 49) of the schemes had stock selection ability, while $69.39 \%$ (34 out of 49) of the schemes did not have stock-selection ability. Further, Carhart Model explained better stock-selection ability among 30.61\% (15 out of 49) schemes. On the other hand $69.39 \%$ (33 out of 49) schemes could not perform better due to poor stock-selection ability of mutual fund managers. Best schemes giving higher risk premium to the investors based on better stock selection ability as per all three models are $28.57 \%$ (14 out of 49) schemes i.e. Birla Sunlife Equity Fund, Birla Sunlife Frontline Equity Fund, Birla Sunlife MNC Fund, DSP BR Opportunities Fund, Franklin India Bluechip Fund, Franklin India Prima Plus, HDFC Equity Fund, HDFC Growth Fund, HDFC Top 200 Fund, HSBC Equity Fund, JM Basic Fund, Relaince Growth Fund, Tata Pure Equity Fund and Sundaram Select Mid-cap Fund.

The present study would be helpful to the investors in making their investment decisions, while parking their money in mutual funds. As informed investors, they will in a better position to select mutual fund schemes having better stock-selection ability over a period of ten years under study. This will enhance the confidence of investors, which will result in increasing the investments in the mutual fund industry.

The study is confined to growth open-ended equity schemes only and can be further extended to other schemes as the stock-selection ability is important from the point of view of all the schemes. More factors can be studiedby the future researchers like liquidity, investment and profitability as research in asset pricing models is a continuous process and stock-selection ability of mutual fund managers can be measured more elaborately. 


\section{References}

1. Ariff \& Johnson. (1990). Unit Trusts and Their performance, Securities \& Stock pricing, Longman Singapore publishers (pvt) ltd.

2. Coggin, T. D., Fabozzi, F. J., \& Rahman, S. (1993). The investment performance of US equity pension fund managers: An empirical investigation. The Journal of Finance, 48(3), 1039-1055.

3. Cole, Joseph and IP, Y.K. (1993). Decomposition of the performance of Investment Funds and its Implications for market Efficiency, Third International conference organized by Department of Finance \&Accounting, National University of Singapore.

4. Cremers, K.M. \& Petajisto, A. (2009). How active is your fund manager? A new measure that predicts performance, Review of Financial Studies, 22(9), 3329-3369.

5. Cumby, R.E. and J.D. Glen. (1990). Evaluating the performance of International Mutual Funds, Journal of Finance 45, 497-521.

6. Daniel, K., Grinblatt, M., Titman, S., \&Wermers, R. (1997). Measuring mutual fund performance with characteristic-based benchmarks, The Journal of finance, 52(3), 1035-1058.

7. Elton, E. J., Gruber, M. J., \& Blake, C. R. (1995). The Persistence of Risk-Adjusted Mutual Fund Performance, The Journal of Finance, 48(2), 978-998.

8. Fama, Eugene F. (1972). Components of Investment performance, Journal of Finance, 27.

9. Fama, E. F., \& French, K. R. (1992). The Cross-section of Expected Stock Returns, The Journal of Finance, 47(2), 427-465.

10. Fama, E. F., \& French, K. R. (1993). Common Risk Factors in the Returns on Stocks and Bonds, Journal of Financial Economics, 33(1), 3-56.

11. Asness, Moskowitz and Pedersen (2013). Value and Momentum Everywhere. The Journal offinance, 68(3).

12. Barua, SK., and Verma, JR. (1991). Master Share: A Bonanza for Large Investors, Vikalpa, Jan- March, 29-34.

13. Carhart, M. M. (1997). On persistence in mutual fund performance. The Journal of finance, 52(1), 5782.

14. Carlson, R. S. (1970). Aggregate performance of mutual funds, 1948-1967. Journal of Financial and Quantitative Analysis, 5(01), 1-32.

15. Chevalier, J., \& Ellison, G. (1999). Are some mutual fund managers better than others? Cross-sectional patterns in behavior and performance. The Journal of Finance, 54(3), 875-899.

16. Friend, Irwin and Vickers, Douglas (1965). Portfolio Selection and Investment Performance, The Journal of Finance, 20(3), 391-415.

17. IP, Y.K., and HO.Y.K.(1989). Evaluation of performance of mutual Funds in Asian Pacific Region. In Proceedings of the Inaugural International Conference on Asian-Pacific Financial Market Organized by Dept. of Finance \& Banking, Nov., Singapore.

18. James R.F. Guy (1978). The performance of the British Investment Trust Industry, The Journal of Finance, 30(2), 443-55.

19. Jayadev, M. (1998). Investment Policy and Performance of Mutual Funds, Kanishka Publishers, and New Delhi.

20. Jensen, M. C. (1968). The Performance of Mutual Funds in the Period 1945-1964, The Journal of Finance, 23(2), 389-416.

21. John G. Mc Donald (1974). Objectives and Performance of Mutual Funds 1960-1967, Journal of Financial and Quantitative Analysis, 311-33.

22. Kaura, M.N., and Jayadev M. (1995). Performance of Growth Oriented Mutual Funds: An Evaluation., The ICFAI Journal of Applied Finance, 1(1), 1-14.

23. Kaura, M N and Jayadev, M. (1999). Performance of Growth Oriented Mutual Funds: An Evaluation, in T. Ravishankar (ed.) Research Papers in Applied Finance, Institute of Chartered.

24. Low, S. W. (2012). Market Timing and Selectivity Performance: A Cross-Sectional Analysis of Malaysian Unit Trust Funds. Prague Economic Papers,2, 205-219. 
Financial Markets, Institutions and Risks, Volume 3, Issue 3, 2019

ISSN (online) - 2521-1242 ISSN (print) - 2521-1250

25. Markowitz, H. (1952). Portfolio Selection. The Journal of Finance, 7(1), 77-91.

26. Richard A. Ippolito, (1993). On Studies of Mutual Fund Performance, 1962-1991, Financial Analysts Journal, 42- 50.

27. Shah, Ajay and Thomas Susan, (1994). Performance in Evaluation of Professional Portfolio Management in India, A paper prepared by CMIE, 10 April.

28. Sharpe, W. F. (1966). Mutual Fund Performance. The Journal of Business,39(1), 119-138.

29. Thiripalraju, M. and P.R. Patil. (1998). Micro and macro forecasting abilities of Indian fund managers', in Indian Capital Markets: Theories and Empirical Evidence, UTI Institute of Capital Markets and Quest Publications, Mumbai. 
Table 5.7. Carhart Model results for Open-ended Growth Equity Schemes

\begin{tabular}{|c|c|c|c|c|c|c|c|c|c|c|c|c|}
\hline S.No. & Name of the Mutual Fund & Adjusted R & Intercept & $t$ Stat & Beta & $t$ Stat & $S M B$ & $t$ Stat & $H M L$ & $t$ Stat & $W M L$ & $t$ Stat \\
\hline 1 & Birla Sunlife Advantage Fund & 0.936 & -0.001 & -0.12 & $0.96^{*}$ & 164.52 & $-0.028^{*}$ & -2.50 & $0.033 *$ & 3.41 & $0.022 *$ & 2.40 \\
\hline 2 & Birla Sunlife Buy India Fund & 0.799 & $0.024 *$ & 2.01 & $0.77 *$ & 89.29 & $0.191 *$ & 11.72 & $0.081 *$ & 5.60 & $-0.029 *$ & -2.17 \\
\hline 3 & Birla Sunlife Dividend Yield Plus & 0.849 & 0.006 & 0.57 & $0.76^{*}$ & 104.76 & $0.119^{*}$ & 8.57 & $0.169^{*}$ & 13.75 & $0.102 *$ & 9.05 \\
\hline 4 & Birla Sunlife Equity Fund & 0.933 & $0.017 *$ & 2.07 & $0.93 *$ & 164.67 & $0.081 *$ & 7.49 & $0.025^{*}$ & 2.64 & $0.018^{*}$ & 2.09 \\
\hline 5 & Birla Sunlife Frontline Equity Fund & 0.953 & $0.034 *$ & 2.00 & $0.86^{*}$ & 195.39 & $-0.035^{*}$ & -4.15 & -0.007 & -0.97 & 0.000 & -0.67 \\
\hline 6 & Birla Sunlife Mid-Cap Fund & 0.862 & 0.013 & 1.21 & $0.87^{*}$ & 113.10 & $0.287^{*}$ & 19.59 & $0.113 *$ & 8.70 & -0.005 & -0.38 \\
\hline 7 & Birla Sunlife MNC Fund & 0.763 & $0.030 *$ & 2.70 & $0.59^{*}$ & 84.80 & $0.061^{*}$ & 4.64 & 0.015 & 1.17 & 0.022 & 1.82 \\
\hline 8 & Birla Sunlife Opportunities Fund & 0.809 & -0.007 & -0.52 & $0.82 *$ & 92.57 & $0.219^{*}$ & 13.02 & $-0.065^{*}$ & -4.37 & $-0.093 *$ & -6.79 \\
\hline 9 & DSP BR Opportunities Fund & 0.960 & $0.017 *$ & 2.83 & $0.89 *$ & 213.24 & $0.018^{*}$ & 2.31 & $0.021^{*}$ & 3.06 & $0.076^{*}$ & 11.69 \\
\hline 10 & DWS Alpha Equity Fund & 0.926 & 0.008 & 0.90 & $0.92 *$ & 154.37 & -0.007 & -0.65 & $-0.043 *$ & -4.27 & $0.103 *$ & 11.17 \\
\hline 11 & Escorts Growth Plan & 0.021 & 0.007 & 0.12 & $0.23 *$ & 5.99 & $0.287^{*}$ & 3.96 & 0.114 & 1.76 & $0.163^{*}$ & 2.74 \\
\hline 12 & Franklin India Bluechip Fund & 0.942 & $0.0290^{*}$ & 4.08 & $0.87 *$ & 24.73 & $-0.04 *$ & -4.79 & $-.083^{*}$ & -9.84 & $.019^{*}$ & 2.46 \\
\hline 13 & Franklin India Prima Fund & 0.828 & 0.006 & 0.54 & $0.85^{*}$ & 99.07 & $0.362 *$ & 21.96 & $0.173 *$ & 12.29 & $0.040^{*}$ & 3.00 \\
\hline 14 & Franklin India Prima Plus & 0.941 & $-0.0038^{*}$ & -22.69 & $0.86^{*}$ & 182.76 & $0.000^{*}$ & 8.33 & $0.000 *$ & -3.01 & $0.00^{*}$ & -0.30 \\
\hline 16 & HDFC Equity Fund & 0.912 & $0.029 *$ & 3.21 & $0.90^{*}$ & 139.81 & $0.026^{*}$ & 2.12 & $0.022^{*}$ & 2.02 & -0.009 & -0.92 \\
\hline 17 & HDFC Growth Fund & 0.928 & $0.021 *$ & 2.75 & $0.86^{*}$ & 158.01 & $0.057^{*}$ & 5.59 & $0.028^{*}$ & 3.11 & $0.035^{*}$ & 4.16 \\
\hline 18 & HDFC Top 200 Fund & 0.953 & $0.034 *$ & 5.13 & $0.90^{*}$ & 191.80 & $-0.069^{*}$ & -7.80 & -0.008 & -1.07 & 0.009 & 1.27 \\
\hline 19 & HSBC Equity Fund & 0.939 & $0.018^{*}$ & 2.52 & $0.88^{*}$ & 171.68 & 0.002 & 0.17 & $-0.033^{*}$ & -3.89 & $0.084 *$ & 10.59 \\
\hline 20 & ICICI Pru Dynamic Plan & 0.097 & 0.032 & 1.21 & $0.26^{*}$ & 13.72 & $0.263^{*}$ & 7.36 & $0.132^{*}$ & 4.19 & $0.161 *$ & 5.50 \\
\hline 21 & ICICI Pru Top 100 & 0.074 & 0.021 & 0.69 & $0.26^{*}$ & 12.08 & $0.203 *$ & 4.98 & $0.120^{*}$ & 3.33 & $0.195^{*}$ & 5.86 \\
\hline 22 & ICICI Pru Top 200 & 0.946 & $0.015^{*}$ & 2.00 & $0.91 *$ & 177.44 & $-0.095 *$ & -9.71 & $-0.056^{*}$ & -6.44 & $0.052 *$ & 6.56 \\
\hline 23 & ING Core Equity Fund & 0.064 & 0.010 & 0.32 & $0.26^{*}$ & 11.33 & $0.238^{*}$ & 5.39 & $0.127 *$ & 3.24 & $0.147 *$ & 4.05 \\
\hline 24 & JM Basic Fund & 0.286 & $0.488^{*}$ & 12.13 & $1.03 *$ & 91.30 & $-0.564 *$ & -14.18 & $0.360^{*}$ & 9.44 & $-0.380 *$ & -10.70 \\
\hline 25 & JM Equity Fund & 0.804 & 0.000 & 0.056 & $1.00^{*}$ & 139.37 & $0.001 *$ & 7.33 & $0.00^{*}$ & 1.96 & $0.00^{*}$ & -7.2 \\
\hline 26 & Kotak 50 & 0.731 & $-0.043 *$ & -2.38 & $0.92 *$ & 72.97 & 0.043 & 1.80 & $-0.064 *$ & -3.02 & $0.133^{*}$ & 6.76 \\
\hline 27 & LIC Nomura Growth Fund & 0.874 & -0.002 & -0.14 & $0.96^{*}$ & 113.22 & -0.004 & -0.24 & -0.002 & -0.16 & -0.009 & -0.72 \\
\hline 28 & LIC Noumra Equity Fund & 0.922 & -0.018 & -1.88 & $1.00^{*}$ & 148.47 & -0.005 & -0.40 & 0.005 & 0.45 & $0.042 *$ & 4.00 \\
\hline 29 & Morgan Stanley Growth Fund & 0.945 & -0.006 & -0.87 & $0.93 *$ & 180.16 & 0.013 & 1.34 & $-0.027 *$ & -3.09 & $0.048 *$ & 6.01 \\
\hline
\end{tabular}


Table 5.7. (cont.). Carhart Model results for Open-ended Growth Equity Schemes

\begin{tabular}{|c|c|c|c|c|c|c|c|c|c|c|c|c|}
\hline 30 & Principal Growth Fund & 0.936 & -0.009 & -1.13 & $0.90^{*}$ & 168.15 & $0.070^{*}$ & 6.96 & $0.022^{*}$ & 2.48 & $0.041^{*}$ & 4.95 \\
\hline 31 & Reliance Vision Fund & 0.925 & 0.014 & 1.70 & $0.89 *$ & 152.81 & $0.022 *$ & 2.03 & $0.047 *$ & 4.83 & $0.022 *$ & 2.44 \\
\hline 32 & Reliance Growth Fund & 0.922 & $0.028^{*}$ & 3.43 & $0.88^{*}$ & 154.10 & $0.209 *$ & 19.21 & $0.120^{*}$ & 12.41 & 0.009 & 0.98 \\
\hline 33 & SBI Contra Fund & 0.693 & -0.010 & -0.49 & $0.93 *$ & 65.71 & $0.086^{*}$ & 3.21 & $0.077^{*}$ & 3.21 & 0.035 & 1.62 \\
\hline 34 & Sundram Growth Fund & 0.940 & 0.001 & 0.17 & $0.95^{*}$ & 171.05 & -0.011 & -1.02 & $0.060^{*}$ & 6.38 & $0.035^{*}$ & 4.02 \\
\hline 35 & Sundram Midcap Fund & 0.859 & $0.0260^{*}$ & 2.546 & $0.856^{*}$ & 115.37 & $0.285^{*}$ & 20.82 & $0.172^{*}$ & 14.28 & $-0.032 *$ & -2.832 \\
\hline 36 & Sundram Select Focus Fund & 0.909 & 0.007 & 0.77 & $0.92 *$ & 135.95 & $-0.048 *$ & -3.74 & 0.016 & 1.44 & $0.059^{*}$ & 5.66 \\
\hline 37 & Tata Bonaza Fund & 0.792 & -0.028 & -1.71 & $1.01 *$ & 87.12 & $0.146^{*}$ & 6.60 & 0.026 & 1.31 & $0.040^{*}$ & 2.24 \\
\hline 38 & Tata Discovery Fund & 0.782 & $-0.043 *$ & -2.47 & $1.07 *$ & 86.16 & $0.403 *$ & 17.14 & $0.199^{*}$ & 9.52 & $0.065^{*}$ & 3.37 \\
\hline 39 & Tata Equity Opportunities Fund & 0.922 & 0.008 & 0.94 & $0.95^{*}$ & 156.17 & $0.203 *$ & 17.51 & $0.038^{*}$ & 3.73 & $0.111^{*}$ & 11.67 \\
\hline 40 & Tata Ethical Fund & 0.883 & 0.005 & 0.44 & $0.92 *$ & 125.71 & $0.239^{*}$ & 17.23 & $0.031^{*}$ & 2.52 & $0.104 *$ & 9.10 \\
\hline 41 & Tata Growth Fund & 0.876 & -0.008 & -0.66 & $0.91 *$ & 118.00 & $0.313^{*}$ & 20.31 & $0.093^{*}$ & 6.56 & $0.035^{*}$ & 2.68 \\
\hline 42 & Tata Pure Equity Fund & 0.936 & $0.020^{*}$ & 2.63 & $0.90^{*}$ & 169.28 & $0.051^{*}$ & 5.08 & -0.013 & -1.41 & $0.100^{*}$ & 12.05 \\
\hline 43 & Tatastarshare Fund & 0.475 & 0.000 & 0.00 & $1.05^{*}$ & 43.21 & $0.287^{*}$ & 6.25 & $0.101 *$ & 2.48 & 0.046 & 1.22 \\
\hline 44 & UTI Equity Fund & 0.873 & 0.002 & 0.17 & $0.82^{*}$ & 115.16 & $0.043^{*}$ & 3.17 & $0.027^{*}$ & 2.24 & $0.075^{*}$ & 6.85 \\
\hline 47 & UTI Master Value Fund & 0.501 & -0.041 & -1.61 & $0.83^{*}$ & 45.78 & $0.270^{*}$ & 7.87 & $0.105^{*}$ & 3.44 & $0.068^{*}$ & 2.42 \\
\hline 48 & UTI MNC Fund & 0.747 & 0.009 & 0.75 & $0.64 *$ & 79.67 & $0.215^{*}$ & 14.21 & 0.017 & 1.27 & $0.079 *$ & 6.40 \\
\hline 49 & UTI Top 100 Fund & 0.472 & -0.046 & -1.51 & $0.90^{*}$ & 41.53 & 0.037 & 0.90 & -0.004 & -0.11 & $0.091 *$ & 2.70 \\
\hline
\end{tabular}

Note: "*” represent significant at $5 \%$. 